\title{
On Constructions of Optical Queues with a Limited Number of Recirculations
}

\author{
Jay Cheng, Cheng-Shang Chang, Tsz-Hsuan Chao, Duan-Shin Lee, and Ching-Min Lien \\ Institute of Communications Engineering \\ National Tsing Hua University \\ Hsinchu 30013, Taiwan, R.O.C. \\ Email: jcheng@ee.nthu.edu.tw, cschang@ee.nthu.edu.tw \\ thchao@gibbs.ee.nthu.edu.tw, lds@cs.nthu.edu.tw \\ keiichi@gibbs.ee.nthu.edu.tw
}

\begin{abstract}
Recently, there has been a lot of attention on the constructions of optical queues by using optical Switches and fiber Delay Lines (SDL). In this paper, we consider the constructions of optical queues with a limited number of recirculations through the fibers in such SDL constructions. Such a limitation on the number of recirculations comes from practical feasibility considerations, such as crosstalk, power loss, amplified spontaneous emission (ASE) from the Erbium doped fiber amplifiers (EDFA), and the pattern effect of the optical switches.

We first transform the design of the fiber delays in such SDL constructions to an equivalent integer representation problem. Specifically, given $1 \leq k \leq M$, we seek for an $M$-sequence $\mathbf{d}_{1}^{M}=\left(d_{1}, d_{2}, \ldots, d_{M}\right)$ of positive integers to maximize the number of consecutive integers (starting from 0 ) that can be represented by the $\mathcal{C}$-transform relative to $d_{1}^{M}$ such that there are at most $k$ 1-entries in their $\mathcal{C}$-transforms. Then we give a class of greedy constructions so that $d_{1}, d_{2}, \ldots, d_{M}$ are obtained recursively and the maximum number of representable consecutive integers by using $d_{1}, d_{2}, \ldots, d_{i}$ is larger than that by using $d_{1}, d_{2}, \ldots, d_{i-1}$ for all $i$. Furthermore, we obtain an explicit recursive expression for $d_{1}, d_{2}, \ldots, d_{M}$ given by a greedy construction. Finally, we show that an optimal $M$-sequence (in the sense of achieving the maximum number of representable consecutive integers) can be given by a greedy construction. The solution of such an integer representation problem can be applied to the construction of optical 2-to-1 FIFO multiplexers with a limited number of recirculations. We show that the complexity of searching for an optimal construction can be greatly reduced from exponential time to polynomial time by only considering the greedy constructions instead of performing an exhaustive search. Similar results can be obtained for linear compressors and linear decompressors with a limited number of recirculations.
\end{abstract}

\section{INTRODUCTION}

It is well recognized that one of the most critically sought after technologies in all-optical packet switching is the constructions of optical queues for contention resolution among packets competing for the same resources in the optical domain. Recently, there has been a lot of attention in the literature (see e.g., [1]-[25] and the references therein) on the constructions of optical queues by using optical Switches and fiber Delay Lines (SDL) to route the optical packets to the right place at the right time so as to achieve exact emulations of the optical queues. These SDL constructions of optical queues include First-In First-Out (FIFO) multiplexers in [4]-[6] and
[11]-[16], buffered packet switches in [6]-[7], FIFO queues in [17]-[19], Last-In First-Out (LIFO) queues in [19], priority queues in [20]-[22], and linear compressors, non-overtaking delay lines, and flexible delay lines in [23]-[24].

However, there are some important practical feasibility issues of concern that need to be addressed in the SDL constructions of optical queues. As pointed out in [26]-[28], crosstalk due to power leakage from other optical links, power loss experienced during recirculations through the optical switches and the fiber delay lines, amplified spontaneous emission (ASE) from the Erbium doped fiber amplifiers (EDFA) that are used for boosting the signal power, and the pattern effect of the optical switches, among others, lead to a limitation on the number of times that an optical packet can be recirculated through the optical switches and the fiber delay lines. If such an issue is not taken into consideration during the design of optical queues, then for an optical packet recirculated through the optical switches and the fiber delay lines for a great number of times, there is a good chance that it can not be reliably recognized at the destined output port due to severe power loss and/or serious noise accumulation even if it appears at the right place and at the right time. As such, SDL constructions of optical queues with a limited number of recirculations through the fibers is a very important practical design issue.

For certain optical queues, including 2-to-1 FIFO multiplexers, linear compressors, and linear decompressors, the delay $x$ of a packet is known upon its arrival and the routing of the packet is according to the $\mathcal{C}$-transform [13] $\mathcal{C}(x)=$ $\left(I_{1}(x), I_{2}(x), \ldots, I_{M}(x)\right)$ (a generalization of the well-known binary representation) of the packet delay $x$ with respect to the $M$-sequence $\mathbf{d}_{1}^{M}=\left(d_{1}, d_{2}, \ldots, d_{M}\right)$ of the delays of the fibers in the queue. For these optical queues, there is a prominent route-once property that says that an optical packet can be routed through each fiber at most once. Specifically, if $I_{i}(x)=1$ for some $1 \leq i \leq M$, then the packet will be routed through the $i^{\text {th }}$ fiber with delay $d_{i}$ once; otherwise, the packet will not be routed to the $i^{\text {th }}$ fiber. For instance, if $I_{i}(x)=1$ for all $i=1,2, \ldots, M$, then the packet will be routed through each of the $M$ fibers once.

The problem arises if there is a limitation on the number 
$k$ of recirculations through the $M$ fibers due to the practical feasibility considerations mentioned above. If $k<M$, then a packet routed through more than $k$ of the $M$ fibers can not be reliably recognized at the destined output port. As such, in such situations the buffer size (for 2-to-1 FIFO multiplexers) or the maximum delay (for linear compressors and linear decompressors) is given by the maximum representable integer $B\left(\mathbf{d}_{1}^{M} ; k\right)$ with respect to $\mathbf{d}_{1}^{M}$ and $k$, namely, the largest positive integer such that each of the nonnegative integers not exceeding it has a $\mathcal{C}$-transform (with respect to $\mathbf{d}_{1}^{M}$ ) with the number of 1-entries less than or equal to $k$. It follows that the problem of constructing the delays $d_{1}, d_{2}, \ldots, d_{M}$ for these optical queues with a limited number $k$ of recirculations through the fibers and with buffer size/maximum delay $B\left(\mathbf{d}_{1}^{M} ; k\right)$ is equivalent to the integer representation problem of constructing an $M$-sequence $\mathbf{d}_{1}^{M}$ with maximum representable integer $B\left(\mathbf{d}_{1}^{M} ; k\right)$ with respect to $\mathbf{d}_{1}^{M}$ and $k$.

Given $1 \leq k \leq M$. Our first contribution in this paper is to give a class of greedy constructions for the $M$-sequence $\mathbf{d}_{1}^{M}$. An $M$-sequence $\mathbf{d}_{1}^{M}$ given by a greedy construction is obtained recursively in a greedy manner so that the maximum representable integer is increased when each $d_{i}$ is added to the already determined $d_{1}, d_{2}, \ldots, d_{i-1}$ for all $i$. Denote the set of the $M$-sequences obtained by such greedy constructions as $\mathcal{G}_{M, k}$. Then we also obtain an explicit recursive expression for an $M$-sequence $\mathbf{d}_{1}^{M} \in \mathcal{G}_{M, k}$ and for the corresponding maximum representable integer $B\left(\mathbf{d}_{1}^{M} ; k\right)$. Our second contribution is to show that the largest possible maximum representable integer $B(M, k)=\max _{\mathbf{d}_{1}^{M} \in \mathcal{A}_{M}} B\left(\mathbf{d}_{1}^{M} ; k\right)$ that can be achieved by an $M$-sequence in $\mathcal{A}_{M}$, where $\mathcal{A}_{M}$ is the set of all $M$-sequences satisfying the condition in (A2) (in Section II) is indeed achieved by an $M$-sequence in $\mathcal{G}_{M, k}$ (it is shown that $\mathcal{G}_{M, k}$ is a subset of $\mathcal{A}_{M}$ in Theorem 5), namely, $B(M, k)=\max _{\mathbf{d}_{1}^{M} \in \mathcal{G}_{M, k}} B\left(\mathbf{d}_{1}^{M} ; k\right)$. This implies that an optimal construction (in the sense of maximizing the buffer size) of an optical 2-to-1 multiplexer with a limited number of recirculations could be given by a greedy construction. As such, the complexity of searching for an optimal construction is greatly reduced by only considering the greedy constructions when compared to performing an exhaustive search (polynomial time vs. exponential time). Similar results can be obtained for optimal constructions of linear compressors and linear decompressors with a limited number of recirculations, and results along this line will be reported separately in the near future.

This paper is organized as follows. In Section II, we describe the transformation of the constructions of certain optical queues into an equivalent integer representation problem in detail. In Section III, we give a class of greedy constructions for the $M$-sequence $\mathbf{d}_{1}^{M}$ in the equivalent integer representation problem, and obtain an explicit recursive expression for such an $M$-sequence $\mathbf{d}_{1}^{M}$ and for the corresponding maximum representable integer $B\left(\mathbf{d}_{1}^{M} ; k\right)$. In Section IV, we show that an optimal construction can be given by a greedy construction. Finally, we conclude this paper in Section V.

\section{The Integer Representation Problem}

We first review the $\mathcal{C}$-transform in [13] for the unique representation of nonnegative integers. The $\mathcal{C}$-transform could be regarded as a generalization of the well-known binary representation for the unique representation of nonnegative integers.

Definition 1 (C-Transform) [13] Given an $M$-sequence $\mathbf{d}_{1}^{M}=\left(d_{1}, d_{2}, \ldots, d_{M}\right)$ of positive integers. The $\mathcal{C}$-transform of a nonnegative integer $x$ with respect to $\mathbf{d}_{1}^{M}$ is defined as the $M$-sequence $\mathcal{C}(x)=\left(I_{1}(x), I_{2}(x), \ldots, I_{M}(x)\right)$, where the entries $I_{M}(x), I_{M-1}(x), \ldots, I_{1}(x)$, in that order, are given recursively by

$$
I_{i}(x)= \begin{cases}1, & \text { if } x-\sum_{j=i+1}^{M} I_{j}(x) d_{j} \geq d_{i}, \\ 0, & \text { otherwise }\end{cases}
$$

Theorem 2 [13] Given an $M$-sequence $\mathbf{d}_{1}^{M}$ of positive integers. The $\mathcal{C}$-transform $\mathcal{C}(x)$ of $x$ with respect to $\mathbf{d}_{1}^{M}$ is the unique representation of $x$ for all $x=0,1, \ldots, \sum_{i=1}^{M} d_{i}$, i.e.,

$$
x=\sum_{i=1}^{M} I_{i}(x) d_{i}, \text { for all } x=0,1, \ldots, \sum_{i=1}^{M} d_{i},
$$

if and only if the following condition in (A1) holds:

(A1) $d_{1}=1$ and $1 \leq d_{i+1} \leq \sum_{j=1}^{i} d_{j}+1$ for $i=$ $1,2, \ldots, M-1$.

In [13], it was shown that under a simple packet routing policy, the feedback system in Figure 1 consisting of an $(M+$ $2) \times(M+2)$ crossbar switch and $M$ fiber delay lines with delays $d_{1} \leq d_{2} \leq \cdots \leq d_{M}$ can be operated as a 2-to-1 FIFO multiplexer with buffer $\sum_{i=1}^{M} d_{i}$ if and only if the following condition in (A2) holds:

(A2) $\quad d_{1}=1$ and $d_{i} \leq d_{i+1} \leq 2 d_{i}$ for $i=1,2, \ldots, M-1$. We note that the condition in (A2) is stronger than the condition in (A1) as it has been shown in [13] that if $d_{1}, d_{2}, \ldots, d_{M}$ satisfy the condition in (A2), then they also satisfy the condition in (A1).

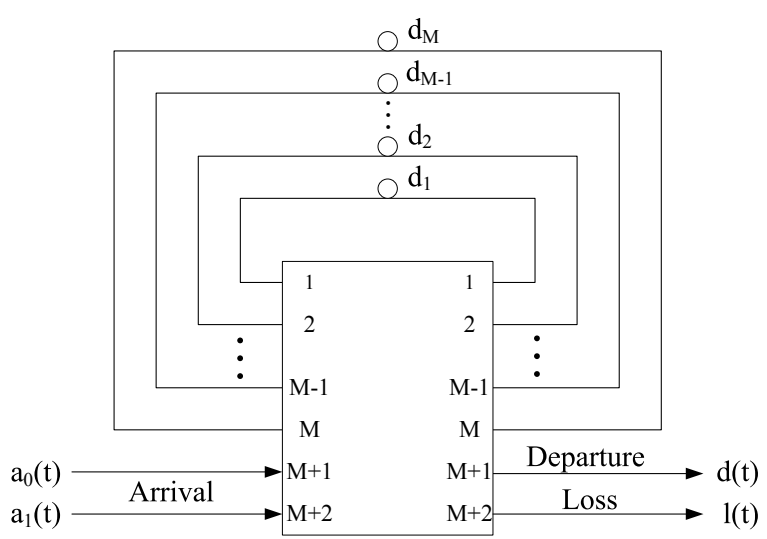

Fig. 1. A 2-to-1 FIFO multiplexer with buffer $\sum_{i=1}^{M} d_{i}$. 
Furthermore, it was shown in [24] that if the condition in (A1) holds, then the construction in Figure 2 by a concatenation of $M$ scaled optical memory cells with scaling factors $d_{1}, d_{2}, \ldots, d_{M}$ (the $i^{\text {th }}$ scaled optical memory cell with scaling factor $d_{i}$ in Figure 2 is built by a $2 \times 2$ crossbar switch and a fiber delay line with delay $d_{i}$ for $i=1,2, \ldots, M$ ) can be operated as a self-routing linear compressor with maximum delay $\sum_{i=1}^{M} d_{i}$.

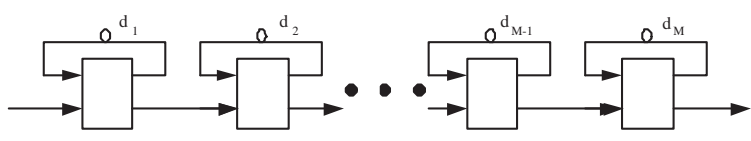

Fig. 2. A construction of a linear compressor with maximum delay $\sum_{i=1}^{M} d_{i}$ by a concatenation of $M$ scaled optical memory cells with scaling factors $d_{1}, d_{2}, \ldots, d_{M}$.

Regarding the self-routing policy for the above two queues, suppose that the delay of a packet arriving at time $t$ is $0 \leq$ $x \leq \sum_{i=1}^{M} d_{i}$ and the expression of $x$ by the $\mathcal{C}$-transform of $x$ is

$$
x=d_{i_{1}}+d_{i_{2}}+\cdots+d_{i_{k}}
$$

where $1 \leq i_{1}<i_{2}<\cdots<i_{k} \leq M$, then the packet is routed to the fiber with delay $d_{i_{1}}$ at time $t$, to the fiber with delay $d_{i_{2}}$ at time $t+d_{i_{1}}, \ldots$, and to the fiber with delay $d_{i_{k}}$ at time $t+\sum_{\ell=1}^{k-1} d_{i_{\ell}}$.

The problem arises if there is a limitation on the number $k$ of recirculations through the $M$ fibers in order to ensure that a packet can be reliably recognized at the destined output port. In such situations, the buffer size (for 2-to-1 FIFO multiplexers) or the maximum delay (for linear compressors and linear decompressors) is given by the largest positive integer such that each of the nonnegative integers not exceeding it has a $\mathcal{C}$-transform with the number of 1 -entries less than or equal to $k$ as a packet with delay one more than this largest positive integer will be routed through more than $k$ fibers and hence can not be reliably recognized at the destined output port. We call such a largest positive integer the maximum representable integer with respect to $\mathbf{d}_{1}^{M}$ and $k$, which is formally defined below.

Definition 3 (Maximum Representable Integer) Given an $M$-sequence $\mathrm{d}_{1}^{M}$ of positive integers and a positive integer $k$. The maximum representable integer $B\left(\mathbf{d}_{1}^{M} ; k\right)$ with respect to $\mathbf{d}_{1}^{M}$ and $k$ is defined as the largest positive integer such that each of the nonnegative integers not exceeding it has a $\mathcal{C}$-transform with the number of 1-entries less than or equal to $k$, i.e.,

$B\left(\mathbf{d}_{1}^{M} ; k\right)=\max \left\{y: \sum_{i=1}^{M} I_{i}(x) \leq k, x=0,1, \ldots, y\right\}$.

For obvious reasons, we also define $B\left(\mathbf{d}_{1}^{M} ; k\right)=0$ if $M=0$ or $k=0$.
Note that from Theorem 2 we have $B\left(\mathbf{d}_{1}^{M} ; k\right) \stackrel{3}{=}$ $B\left(\mathbf{d}_{1}^{M} ; M\right)=\sum_{\ell=1}^{M} d_{\ell}$ for $k \geq M$ if $\mathbf{d}_{1}^{M}$ satisfies the condition in (A1).

As we are most interested in the constructions of these optical queues with as large buffer size/maximum delay as possible, the optimal constructions (in the sense of maximizing the buffer size/maximum delay) of these optical queues with a limited number of recirculations through the fibers is equivalent to the integer representation problem of constructing an $M$-sequence that achieves the largest possible maximum representable integer.

In this paper, we focus on a class of greedy constructions of the $M$-sequence $\mathbf{d}_{1}^{M}$ in Section III, and show that an $M$-sequence achieving the largest possible maximum representable integer $B\left(\mathbf{d}_{1}^{M} ; k\right)$ over all $\mathbf{d}_{1}^{M} \in \mathcal{A}_{M}$, where $\mathcal{A}_{M}$ is the set of all $M$-sequences satisfying the condition in (A2), could be given by a greedy construction in Section IV. The results in this paper can therefore be applied to the optimal constructions of 2-to-1 FIFO multiplexers with a limited number of recirculations through the fibers. We note that similar results can be obtained if the maximization of the maximum representable integer $B\left(\mathbf{d}_{1}^{M} ; k\right)$ is over all $M$ sequences satisfying the condition in (A1), and the results can be applied to the optimal constructions of linear compressors and linear decompressors with a limited number of recirculations through the fibers.

\section{A Class of Greedy Constructions}

Suppose that $1 \leq k \leq M$. In this section, we give a class of greedy constructions of the $M$-sequence $\mathbf{d}_{1}^{M}$, and obtain an explicit recursive expression for $\mathbf{d}_{1}^{M}$ and for the corresponding maximum representable integer $B\left(\mathbf{d}_{1}^{M} ; k\right)$.

Consider the case with $M=6$ and $k=2$. A direct construction of $d_{1}, d_{2}, d_{3}, d_{4}, d_{5}, d_{6}$ is to divide the construction into two parts, the construction of $d_{1}, d_{2}, d_{3}$ and the construction of $d_{4}, d_{5}, d_{6}$, so that there is at most one nonzero entry in $\left\{I_{1}(x), I_{2}(x), I_{3}(x)\right\}$ and there is at most one nonzero entry in $\left\{I_{4}(x), I_{5}(x), I_{6}(x)\right\}$ for as many consecutive nonnegative integers $x$ as possible. For example, we can choose $d_{1}=$ $1, d_{2}=2, d_{3}=3$ and achieve $B\left(\mathbf{d}_{1}^{3} ; 1\right)=3$. Then we can choose $d_{4}=B\left(\mathbf{d}_{1}^{3} ; 1\right)+1=4, d_{5}=2\left(B\left(\mathbf{d}_{1}^{3} ; 1\right)+1\right)=$ $8, d_{6}=3\left(B\left(\mathbf{d}_{1}^{3} ; 1\right)+1\right)=12$. For $4 \leq i \leq 6$, it is easy to see that for $x=d_{i}, d_{i}+1, \ldots, d_{i}+B\left(\mathbf{d}_{1}^{3} ; 1\right)$, there is at most one nonzero entry in $\left\{I_{1}(x), I_{2}(x), I_{3}(x)\right\}$ and there is exactly one nonzero entry in $\left\{I_{4}(x), I_{5}(x), I_{6}(x)\right\}$ (specifically, $I_{i}(x)=1$ ). It follows that such a construction guarantees that $B\left(\mathbf{d}_{1}^{6} ; 2\right) \geq d_{6}+B\left(\mathbf{d}_{1}^{3} ; 1\right)=15$. Indeed, from Table I we see that $B\left(\mathbf{d}_{1}^{6} ; 2\right)=16$, which is larger than 15 .

A better construction, called a greedy construction in this paper, can be described as follows. We still divide the construction into two parts as in the direct construction above. For the construction of $d_{1}, d_{2}, d_{3}$, each $d_{i}$ is constructed recursively by using $d_{1}, d_{2}, \ldots, d_{i-1}$ that have already been determined so that $B\left(\mathbf{d}_{1}^{i} ; 1\right)$ is at least one more than $B\left(\mathbf{d}_{1}^{i-1} ; 1\right)$, and this is possible by choosing $d_{i}=B\left(\mathbf{d}_{1}^{i-1} ; 1\right)+1$ for $i=1,2,3$. 


\begin{tabular}{|c||c|c|c||c|c|c|}
\hline$x$ & $I_{1}(x)$ & $I_{2}(x)$ & $I_{3}(x)$ & $I_{4}(x)$ & $I_{5}(x)$ & $I_{6}(x)$ \\
\hline 0 & 0 & 0 & 0 & 0 & 0 & 0 \\
\hline 1 & 1 & 0 & 0 & 0 & 0 & 0 \\
\hline 2 & 0 & 1 & 0 & 0 & 0 & 0 \\
\hline 3 & 0 & 0 & 1 & 0 & 0 & 0 \\
\hline 4 & 0 & 0 & 0 & 1 & 0 & 0 \\
\hline 5 & 1 & 0 & 0 & 1 & 0 & 0 \\
\hline 6 & 0 & 1 & 0 & 1 & 0 & 0 \\
\hline 7 & 0 & 0 & 1 & 1 & 0 & 0 \\
\hline 8 & 0 & 0 & 0 & 0 & 1 & 0 \\
\hline 9 & 1 & 0 & 0 & 0 & 1 & 0 \\
\hline 10 & 0 & 1 & 0 & 0 & 1 & 0 \\
\hline 11 & 0 & 0 & 1 & 0 & 1 & 0 \\
\hline 12 & 0 & 0 & 0 & 0 & 0 & 1 \\
\hline 13 & 1 & 0 & 0 & 0 & 0 & 1 \\
\hline 14 & 0 & 1 & 0 & 0 & 0 & 1 \\
\hline 15 & 0 & 0 & 1 & 0 & 0 & 1 \\
\hline 16 & 0 & 0 & 0 & 1 & 0 & 1 \\
\hline
\end{tabular}

TABLE I

THE $\mathcal{C}$-TRANSFORM OF $x$ WITH RESPECT TO $\mathbf{d}_{1}^{6}=(1,2,3,4,8,12)$ FOR $x=0,1,2, \ldots, 16$

As such, $d_{1}=B\left(\mathbf{d}_{1}^{0} ; 1\right)+1=1, d_{2}=B\left(\mathbf{d}_{1}^{1} ; 1\right)+1=2, d_{3}=$ $B\left(\mathbf{d}_{1}^{2} ; 1\right)+1=3$. As for the construction of $d_{4}, d_{5}, d_{6}$, each $d_{i}$ is also constructed recursively by using $d_{1}, d_{2}, \ldots, d_{i-1}$ that have already been determined, but now $B\left(\mathbf{d}_{1}^{i} ; 2\right)$ is required to be at least one more than $B\left(\mathbf{d}_{1}^{i-1} ; 2\right)$. Again, this is possible by choosing $d_{i}=B\left(\mathbf{d}_{1}^{i-1} ; 2\right)+1$ for $i=4,5,6$. It follows that $d_{4}=B\left(\mathbf{d}_{1}^{3} ; 2\right)+1=6, d_{5}=B\left(\mathbf{d}_{1}^{4} ; 2\right)+1=10, d_{6}=$ $B\left(\mathbf{d}_{1}^{5} ; 2\right)+1=14$. From Table II, we see that $B\left(\mathbf{d}_{1}^{6} ; 2\right)=17$, which is larger than 16 in the direct construction above.

\begin{tabular}{|c||c|c|c||c|c|c|}
\hline$x$ & $I_{1}(x)$ & $I_{2}(x)$ & $I_{3}(x)$ & $I_{4}(x)$ & $I_{5}(x)$ & $I_{6}(x)$ \\
\hline 0 & 0 & 0 & 0 & 0 & 0 & 0 \\
\hline 1 & 1 & 0 & 0 & 0 & 0 & 0 \\
\hline 2 & 0 & 1 & 0 & 0 & 0 & 0 \\
\hline 3 & 0 & 0 & 1 & 0 & 0 & 0 \\
\hline 4 & 1 & 0 & 1 & 0 & 0 & 0 \\
\hline 5 & 0 & 1 & 1 & 0 & 0 & 0 \\
\hline 6 & 0 & 0 & 0 & 1 & 0 & 0 \\
\hline 7 & 1 & 0 & 0 & 1 & 0 & 0 \\
\hline 8 & 0 & 1 & 0 & 1 & 0 & 0 \\
\hline 9 & 0 & 0 & 1 & 1 & 0 & 0 \\
\hline 10 & 0 & 0 & 0 & 0 & 1 & 0 \\
\hline 11 & 1 & 0 & 0 & 0 & 1 & 0 \\
\hline 12 & 0 & 1 & 0 & 0 & 1 & 0 \\
\hline 13 & 0 & 0 & 1 & 0 & 1 & 0 \\
\hline 14 & 0 & 0 & 0 & 0 & 0 & 1 \\
\hline 15 & 1 & 0 & 0 & 0 & 0 & 1 \\
\hline 16 & 0 & 1 & 0 & 0 & 0 & 1 \\
\hline 17 & 0 & 0 & 1 & 0 & 0 & 1 \\
\hline
\end{tabular}

TABLE II

THE $\mathcal{C}$-TRANSFORM OF $x$ WITH RESPECT TO $\mathbf{d}_{1}^{6}=(1,2,3,6,10,14)$ FOR $x=0,1,2, \ldots, 17$.

We can now describe our greedy constructions in a general setting. For $M \geq 1$ and $1 \leq k \leq M$, let $n_{1}, n_{2}, \ldots, n_{k}$ be positive integers such that $\sum_{i=1}^{k} n_{i}=M$, and let $s_{0}=0$ and $s_{i}=\sum_{\ell=1}^{i} n_{\ell}$ for $i=1,2, \ldots, k$. Define a sequence of positive integers $d_{1}, d_{2}, \ldots, d_{M}$ recursively as follows:

$$
d_{s_{i}+j}=B\left(\mathbf{d}_{1}^{s_{i}+j-1} ; i+1\right)+1,
$$

where $0 \leq i \leq k-1,1 \leq j \leq n_{i+1}$. In other words, we divide the construction into $k$ parts. For the $(i+1)^{\text {th }}$ part, where $0 \leq i \leq k-1, d_{s_{i}+j}$ is constructed recursively by using $d_{1}, d_{2}, \ldots, d_{s_{i}+j-1}$ according to (5) for $j=1,2, \ldots, n_{i+1}$. We denote $\mathcal{G}_{M, k}$ as the set of all $M$-sequences $\mathbf{d}_{1}^{M}$ given by (5) by using sequences of positive integers $n_{1}, n_{2}, \ldots, n_{k}$ such that $\sum_{i=1}^{k} n_{i}=M$.

After $d_{1}, d_{2}, \ldots, d_{s_{i}+j}$ have been determined, the integers $0,1, \ldots, B\left(\mathbf{d}_{1}^{s_{i}+j-1} ; i+1\right)$ are representable by using at most $i+1$ of the integers $d_{1}, d_{2}, \ldots, d_{s_{i}+j-1}$ according to the $\mathcal{C}$-transform (note that $d_{s_{i}+j}>B\left(\mathbf{d}_{1}^{s_{i}+j-1} ; i+1\right)$ and hence $d_{s_{i}+j}$ is not used in their representations). As we define $d_{s_{i}+j}=B\left(\mathbf{d}_{1}^{s_{i}+j-1} ; i+1\right)+1$ in (5), the integer $B\left(\mathbf{d}_{1}^{s_{i}+j-1} ; i+1\right)+1$ is representable by using $d_{s_{i}+j}$. If we choose $d_{s_{i}+j}>B\left(\mathbf{d}_{1}^{s_{i}+j-1} ; i+1\right)+1$, then the integer $B\left(\mathbf{d}_{1}^{s_{i}+j-1} ; i+1\right)+1$ is not representable by using at most $i+1$ of the integers $d_{1}, d_{2}, \ldots, d_{s_{i}+j}$ according to the $\mathcal{C}$-transform, and such a choice of $d_{s_{i}+j}$ will have no use in increasing the maximum representable integer. This is why we define $d_{s_{i}+j}=B\left(\mathbf{d}_{1}^{s_{i}+j-1} ; i+1\right)+1$ in (5). Furthermore, as $d_{s_{i}+j}$ is used in the representation of the integers not less than $d_{s_{i}+j}$ and the integers $0,1, \ldots, B\left(\mathbf{d}_{1}^{s_{i}+j-1} ; i\right)$ are representable by using at most $i$ of the integers $d_{1}, d_{2}, \ldots, d_{s_{i}+j-1}$, it is clear that the integers $d_{s_{i}+j}, d_{s_{i}+j}+1, \ldots, d_{s_{i}+j}+B\left(\mathbf{d}_{1}^{s_{i}+j-1} ; i\right)$ are representable by using at most $i+1$ of the integers $d_{1}, d_{2}, \ldots, d_{s_{i}+j}$. As such, our construction guarantees that $B\left(\mathbf{d}_{1}^{s_{i}+j} ; i+1\right) \geq d_{s_{i}+j}+B\left(\mathbf{d}_{1}^{s_{i}+j-1} ; i\right)$. Indeed, in Lemma 7 below, we show that $B\left(\mathbf{d}_{1}^{s_{i}+j} ; i+1\right)=d_{s_{i}+j}+B\left(\mathbf{d}_{1}^{s_{i}+j-1} ; i\right)$, i.e., the maximum representable integer is increased by $B\left(\mathbf{d}_{1}^{s_{i}+j} ; i+1\right)-B\left(\mathbf{d}_{1}^{s_{i}+j-1} ; i+1\right)=B\left(\mathbf{d}_{1}^{s_{i}+j-1} ; i\right)+1$.

We call such a construction a greedy construction. Intuitively, it is expected that such greedy constructions possess certain optimal properties. Indeed, in Theorem 9 (in Section IV) we will show that every optimal construction (in the sense of achieving the largest possible maximum representable integer) is a greedy construction.

Note that for $M=1$, we have $d_{1}=d_{s_{0}+1}=B\left(\mathbf{d}_{1}^{0} ; 1\right)+1=$ 1 as $B\left(\mathbf{d}_{1}^{0} ; 1\right)=0$ by definition. For $M \geq 2$ and $k=M$, we must have $n_{1}=n_{2}=\cdots=n_{M}=1$ and $s_{1}=1, s_{2}=$ $2, \ldots, s_{M}=M$. As it is easy to see that $B(1,2, \ldots, n ; 1)=n$ for all $n \geq 1$, it follows from (5) that

$$
\begin{gathered}
d_{1}=d_{s_{0}+1}=B\left(\mathbf{d}_{1}^{0} ; 1\right)+1=1, \\
d_{2}=d_{s_{1}+1}=B\left(\mathbf{d}_{1}^{1} ; 2\right)+1=d_{1}+1=2, \\
d_{3}=d_{s_{2}+1}=B\left(\mathbf{d}_{1}^{2} ; 3\right)+1=\sum_{\ell=1}^{2} d_{\ell}+1=2^{2}, \\
\vdots \\
d_{M}=d_{s_{M-1}+1}=B\left(\mathbf{d}_{1}^{M-1} ; M\right)+1 \\
=\sum_{\ell=1}^{M-1} d_{\ell}+1=2^{M-1},
\end{gathered}
$$


where $d_{1}=1, d_{2}=2, \ldots, d_{i}=2^{i-1}$ satisfy the condition in (A2) for each $i=1,2, \ldots, M-1$, and we have used the fact that $B\left(\mathbf{d}_{1}^{i} ; i+1\right)=B\left(\mathbf{d}_{1}^{i} ; i\right)=\sum_{\ell=1}^{i} d_{\ell}$. As such, in the following we only consider the nontrivial case with $M \geq 2$ and $1 \leq k \leq M-1$. In such a nontrivial case, there must exist some $1 \leq i \leq k$ such that $n_{i} \geq 2$ as otherwise we will have $n_{1}=n_{2}=\cdots=n_{k}=1$ and $\sum_{i=1}^{k} n_{i}=k \leq$ $M-1$, contradicting to $\sum_{i=1}^{k} n_{i}=M$. Furthermore, from the following theorem, it suffices to consider only the case with $n_{1} \geq 2$.

Theorem 4 For $M \geq 2$ and $2 \leq k \leq M-1$, let $n_{1}, n_{2}, \ldots, n_{k}$ be positive integers such that $n_{1}=1$ and $\sum_{i=1}^{k} n_{i}=$ M. Let $i^{\prime}=\min \left\{2 \leq i \leq k: n_{i} \geq 2\right\}$ (note that $i^{\prime}$ is well defined as $n_{1}=1$ and hence there must exist some $2 \leq i \leq k$ such that $n_{i} \geq 2$ ), and let $m_{1}=2, m_{2}=m_{3}=\cdots=m_{i^{\prime}-1}=1, m_{i^{\prime}}=n_{i^{\prime}}-1$, $m_{i^{\prime}+1}=n_{i^{\prime}+1}, m_{i^{\prime}+2}=n_{i^{\prime}+2}, \ldots, m_{k}=n_{k}$. Suppose that $d_{1}, d_{2}, \ldots, d_{M}$ are generated by $n_{1}, n_{2}, \ldots, n_{k}$ by using (5), and $h_{1}, h_{2}, \ldots, h_{M}$ are generated by $m_{1}, m_{2}, \ldots, m_{k}$ by using (5). Then $d_{\ell}=h_{\ell}$ for all $\ell=1,2, \ldots, M$.

The proof of Theorem 4 is omitted due to the space limit (for proof, see [29]).

In the following theorem, we derive an explicit recursive expression for the $M$-sequence $\mathbf{d}_{1}^{M}$ given by (5) by using positive integers $n_{1}, n_{2}, \ldots, n_{k}$ with $\sum_{i=1}^{k} n_{i}=M$, and for the corresponding maximum representable integer $B\left(\mathbf{d}_{1}^{M} ; k\right)$.

Theorem 5 For $M \geq 2$ and $1 \leq k \leq M-1$, let $n_{1}, n_{2}, \ldots, n_{k}$ be positive integers such that $n_{1} \geq 2$ and $\sum_{i=1}^{k} n_{i}=M$, and let $s_{0}=0$ and $s_{i}=\sum_{\ell=1}^{i} n_{\ell}$ for $i=1,2, \ldots, k$. The $M$-sequence $\mathbf{d}_{1}^{M}$ given by (5) can be expressed recursively as follows:

$$
\begin{aligned}
d_{j} & =j, 1 \leq j \leq s_{1}, \\
d_{s_{i}+j} & =2 d_{s_{i}}+(j-1)\left(d_{s_{1}}+d_{s_{2}}+\cdots+d_{s_{i}}+1\right), \\
& 1 \leq i \leq k-1,1 \leq j \leq n_{i+1} .
\end{aligned}
$$

Furthermore, the $M$-sequence $\mathbf{d}_{1}^{M}$ satisfies the condition in (A2), and we have

$$
\begin{aligned}
B\left(\mathbf{d}_{1}^{j} ; 1\right)= & j, 1 \leq j \leq s_{1}, \\
B\left(\mathbf{d}_{1}^{s_{i}+j} ; i+1\right)= & d_{s_{i}+j}+d_{s_{1}}+d_{s_{2}}+\cdots+d_{s_{i}}, \\
& 1 \leq i \leq k-1,1 \leq j \leq n_{i+1} .
\end{aligned}
$$

Theorem 5 tells us that if the $M$-sequence $\mathbf{d}_{1}^{M}$ in Figure 1 is given by (5), then it satisfies the condition in (A2) and hence the feedback system in Figure 1 can be operated as a 2-to-1 FIFO multiplexer with buffer $B\left(\mathbf{d}_{1}^{M} ; k\right)=\sum_{i=1}^{k} d_{s_{i}}$ under the constraint that each packet can be routed through at most $k$ of the $M$ fibers by using the packet routing policy in [13].

We need the following three lemmas for the proof of Theorem 5. Due to space limit, their proofs are omitted but can be found in [29].
Lemma 6 Let $d_{1}, d_{2}, \ldots, d_{n}$ be a sequence of positive integers satisfying the condition in (A2). Suppose that $i \geq 0$ and $\ell^{\prime}=\max \left\{1 \leq \ell \leq n: d_{\ell} \leq B\left(\mathbf{d}_{1}^{n} ; i+1\right)\right\}$, namely, $d_{\ell^{\prime}} \leq B\left(\mathbf{d}_{1}^{n} ; i+1\right)<d_{\ell^{\prime}+1}$ if $1 \leq \ell^{\prime} \leq n-1$, and $B\left(\mathbf{d}_{1}^{n} ; i+1\right) \geq d_{n}$ if $\ell^{\prime}=n$. Then we have

$$
\begin{aligned}
B\left(\mathbf{d}_{1}^{n} ; i+1\right) & =B\left(\mathbf{d}_{1}^{n-1} ; i+1\right)=\cdots=B\left(\mathbf{d}_{1}^{\ell^{\prime}} ; i+1\right) \\
& =d_{\ell^{\prime}}+B\left(\mathbf{d}_{1}^{\ell^{\prime}-1} ; i\right) .
\end{aligned}
$$

We remark that the condition that $\ell^{\prime}=\max \{1 \leq \ell \leq n$ : $\left.d_{\ell} \leq B\left(\mathbf{d}_{1}^{n} ; i+1\right)\right\}$ is essential for the relation $B\left(\mathbf{d}_{1}^{n} ; i+1\right)=$ $d_{\ell^{\prime}}+B\left(\mathbf{d}_{1}^{\ell^{\prime}-1} ; i\right)$ in Lemma 6 to hold. If $B\left(\mathbf{d}_{1}^{n} ; i+1\right)<d_{\ell^{\prime}}$, then obviously it is impossible that $B\left(\mathbf{d}_{1}^{n} ; i+1\right)=d_{\ell^{\prime}}+$ $B\left(\mathbf{d}_{1}^{\ell^{\prime}-1} ; i\right)$. So at least we need the condition that $B\left(\mathbf{d}_{1}^{n} ; i+\right.$ $1) \geq d_{\ell^{\prime}}$. Even if $B\left(\mathbf{d}_{1}^{n} ; i+1\right) \geq d_{\ell^{\prime}}$, it is not always true that $B\left(\mathbf{d}_{1}^{n} ; i+1\right)=d_{\ell^{\prime}}+B\left(\mathbf{d}_{1}^{\ell^{\prime}-1} ; i\right)$ as can be seen from the following counterexample: for $d_{1}=1, d_{2}=2, d_{3}=4$, and $d_{4}=8$, we have $B\left(\mathbf{d}_{1}^{4} ; 2\right)=6 \geq d_{2}$ and $B\left(\mathbf{d}_{1}^{1} ; 1\right)=1$, but $B\left(\mathbf{d}_{1}^{4} ; 2\right) \neq d_{2}+B\left(\mathbf{d}_{1}^{1} ; 1\right)$; however, we have $d_{3} \leq B\left(\mathbf{d}_{1}^{4} ; 2\right)<$ $d_{4}$ and $B\left(\mathbf{d}_{1}^{2} ; 1\right)=2$, and hence $B\left(\mathbf{d}_{1}^{4} ; 2\right)=d_{3}+B\left(\mathbf{d}_{1}^{2} ; 1\right)$.

Lemma 7 For $M \geq 2$ and $1 \leq k \leq M-1$, let $n_{1}, n_{2}, \ldots, n_{k}$ be positive integers such that $n_{1} \geq 2$ and $\sum_{i=1}^{k} n_{i}=M$, and let $s_{0}=0$ and $s_{i}=\sum_{\ell=1}^{i} n_{\ell}$ for $i=1,2, \ldots, k$. Suppose that for some $1 \leq i \leq k-1$ and $0 \leq j \leq n_{i+1}$, the sequence $d_{1}, d_{2}, \ldots, d_{s_{i}+j}$ is given by (6) and (7). Then the sequence $d_{1}, d_{2}, \ldots, d_{s_{i}+j}$ satisfies the condition in (A2), and we have

$$
B\left(\mathbf{d}_{1}^{s_{i}+j} ; i+1\right)=d_{s_{i}+j}+B\left(\mathbf{d}_{1}^{s_{i}+j-1} ; i\right) .
$$

Lemma 8 For $M \geq 2$ and $1 \leq k \leq M-1$, let $n_{1}, n_{2}, \ldots, n_{k}$ be positive integers such that $n_{1} \geq 2$ and $\sum_{i=1}^{k} n_{i}=M$, and let $s_{0}=0$ and $s_{i}=\sum_{\ell=1}^{i} n_{\ell}$ for $i=1,2, \ldots, k$. Suppose that for some $1 \leq i \leq k-1$ and $0 \leq j \leq n_{i+1}-1$, the sequence $d_{1}, d_{2}, \ldots, d_{s_{i}+j}$ is given by (6) and (7), and suppose that

$$
B\left(\mathbf{d}_{1}^{s_{i}} ; i\right)=d_{s_{1}}+d_{s_{2}}+\cdots+d_{s_{i}} .
$$

Then we have

$$
B\left(\mathbf{d}_{1}^{s_{i}+j} ; i\right)=B\left(\mathbf{d}_{1}^{s_{i}} ; i\right) .
$$

Proof. (Proof of Theorem 5) Clearly, it follows from (5) that

$$
\begin{aligned}
& d_{1}=d_{s_{0}+1}=B\left(\mathbf{d}_{1}^{0} ; 1\right)+1=0+1=1, \\
& d_{2}=d_{s_{0}+2}=B\left(\mathbf{d}_{1}^{1} ; 1\right)+1=1+1=2, \\
& d_{3}=d_{s_{0}+3}=B\left(\mathbf{d}_{1}^{2} ; 1\right)+1=2+1=3, \\
& \quad \vdots \\
& d_{s_{1}}=d_{s_{0}+s_{1}}=B\left(\mathbf{d}_{1}^{s_{1}-1} ; 1\right)+1=\left(s_{1}-1\right)+1=s_{1},
\end{aligned}
$$

and

$$
B\left(\mathbf{d}_{1}^{s_{1}} ; 1\right)=s_{1} .
$$

For each $j=1,2, \ldots, s_{1}$, it is easy to see that the sequence $d_{1}=1, d_{2}=2, \ldots, d_{j}=j$ satisfies the condition in (A2). Therefore, we have proved that (6) and (8) hold.

In the following, we show by induction that (7) and (9) hold, and the sequence $d_{1}, d_{2}, \ldots, d_{s_{i}+j}$ satisfies the condition in 
(A2) for all $1 \leq i \leq k-1$ and $1 \leq j \leq n_{i+1}$. From (5) with $i=1$ and $j=1$, and from (11) with $i=1$ and $j=0$, we have

$$
\begin{aligned}
d_{s_{1}+1} & =B\left(\mathbf{d}_{1}^{s_{1}} ; 2\right)+1 \\
& =d_{s_{1}}+B\left(\mathbf{d}_{1}^{s_{1}-1} ; 1\right)+1 \\
& =d_{s_{1}}+\left(d_{s_{1}}-1\right)+1 \\
& =2 d_{s_{1}},
\end{aligned}
$$

where $B\left(\mathbf{d}_{1}^{s_{1}-1} ; 1\right)=s_{1}-1=d_{s_{1}}-1$ follows from (8) and (6). Therefore, (7) holds for $i=1$ and $j=1$. It then follows from Lemma 7 that the sequence $d_{1}, d_{2}, \ldots, d_{s_{1}+1}$ satisfies the condition in (A2), and

$$
B\left(\mathbf{d}_{1}^{s_{1}+1} ; 2\right)=d_{s_{1}+1}+B\left(\mathbf{d}_{1}^{s_{1}} ; 1\right)=d_{s_{1}+1}+d_{s_{1}},
$$

where $B\left(\mathbf{d}_{1}^{s_{1}} ; 1\right)=s_{1}=d_{s_{1}}$ follows from (8) and (6). As such, (9) also holds for $i=1$ and $j=1$.

Now assume as the induction hypothesis that (7) and (9) hold up to some $1 \leq i \leq k-1$ and $1 \leq j \leq n_{i+1}$, where $s_{i}+j<M$, and the sequence $d_{1}, d_{2}, \ldots, d_{s_{i}+j}$ satisfies the condition in (A2). We need to consider the following two cases:

Case $1.1 \leq j \leq n_{i+1}-1$ : In this case, we have $2 \leq j+1 \leq$ $n_{i+1}$. It follows from (5) and the induction hypothesis that

$$
\begin{aligned}
d_{s_{i}+j+1}= & B\left(\mathbf{d}_{1}^{s_{i}+j} ; i+1\right)+1 \\
= & d_{s_{i}+j}+d_{s_{1}}+d_{s_{2}}+\cdots+d_{s_{i}}+1 \\
= & 2 d_{s_{i}}+(j-1)\left(d_{s_{1}}+d_{s_{2}}+\cdots+d_{s_{i}}+1\right) \\
& +d_{s_{1}}+d_{s_{2}}+\cdots+d_{s_{i}}+1 \\
= & 2 d_{s_{i}}+j\left(d_{s_{1}}+d_{s_{2}}+\cdots+d_{s_{i}}+1\right) .
\end{aligned}
$$

We then have from Lemma 7 that the sequence $d_{1}, d_{2}, \ldots, d_{s_{i}+j+1}$ satisfies the condition in (A2), and

$$
B\left(\mathbf{d}_{1}^{s_{i}+j+1} ; i+1\right)=d_{s_{i}+j+1}+B\left(\mathbf{d}_{1}^{s_{i}+j} ; i\right) .
$$

As it is easy to see from the induction hypothesis that (12) holds up to $i$, it follows from (14), $1 \leq i \leq k-1,1 \leq j \leq$ $n_{i+1}-1$, and Lemma 8 that

$$
\begin{aligned}
B\left(\mathbf{d}_{1}^{s_{i}+j+1} ; i+1\right) & =d_{s_{i}+j+1}+B\left(\mathbf{d}_{1}^{s_{i}+j} ; i\right) \\
& =d_{s_{i}+j+1}+B\left(\mathbf{d}_{1}^{s_{i}} ; i\right) \\
& =d_{s_{i}+j+1}+d_{s_{1}}+d_{s_{2}}+\cdots+d_{s_{i}} .
\end{aligned}
$$

Case 2. $j=n_{i+1}$ : Note that as we assume that $s_{i}+j=$ $s_{i+1}<M$, we have $i \leq k-2$ in this case. In this case, we have $s_{i}+j+1=s_{i+1}+1$, and it follows from (5), the induction hypothesis, and Lemma 7 that

$$
\begin{aligned}
d_{s_{i+1}+1} & =B\left(\mathbf{d}_{1}^{s_{i+1}} ; i+2\right)+1 \\
& =d_{s_{i+1}}+B\left(\mathbf{d}_{1}^{s_{i+1}-1} ; i+1\right)+1 .
\end{aligned}
$$

If $n_{i+1}=1$, then $s_{i+1}-1=s_{i}$. As such, we have from the induction hypothesis, Lemma 7, and (5) that

$$
\begin{aligned}
B\left(\mathbf{d}_{1}^{s_{i+1}-1} ; i+1\right)+1 & =B\left(\mathbf{d}_{1}^{s_{i}} ; i+1\right)+1 \\
& =d_{s_{i}}+B\left(\mathbf{d}_{1}^{s_{i}-1} ; i\right)+1 \\
& =d_{s_{i}}+\left(d_{s_{i}}-1\right)+1 \\
& =2 d_{s_{i}} .
\end{aligned}
$$

As we also have $d_{s_{i}+1}=2 d_{s_{i}}$ from the induction hypothesis, it follows from (16) and $s_{i+1}=s_{i}+1$ that

$$
B\left(\mathbf{d}_{1}^{s_{i+1}-1} ; i+1\right)+1=2 d_{s_{i}}=d_{s_{i}+1}=d_{s_{i+1}} .
$$

On the other hand, if $n_{i+1} \geq 2$, then $n_{i+1}-1 \geq 1$ and from the induction hypothesis we have

$$
\begin{aligned}
& B\left(\mathbf{d}_{1}^{s_{i+1}-1} ; i+1\right)+1 \\
= & B\left(\mathbf{d}_{1}^{s_{i}+n_{i+1}-1} ; i+1\right)+1 \\
= & d_{s_{i}+n_{i+1}-1}+d_{s_{1}}+d_{s_{2}}+\cdots+d_{s_{i}}+1 \\
= & 2 d_{s_{i}}+\left(n_{i+1}-2\right)\left(d_{s_{1}}+d_{s_{2}}+\cdots+d_{s_{i}}+1\right) \\
& +d_{s_{1}}+d_{s_{2}}+\cdots+d_{s_{i}}+1 \\
= & 2 d_{s_{i}}+\left(n_{i+1}-1\right)\left(d_{s_{1}}+d_{s_{2}}+\cdots+d_{s_{i}}+1\right) . \\
= & d_{s_{i}+n_{i+1}}=d_{s_{i+1}} .
\end{aligned}
$$

By combining (15), (17), and (18), we have

$$
d_{s_{i+1}+1}=d_{s_{i+1}}+B\left(\mathbf{d}_{1}^{s_{i+1}-1} ; i+1\right)+1=2 d_{s_{i+1}},
$$

which is the desired result.

Again, we have from Lemma 7 that the sequence $d_{1}, d_{2}, \ldots, d_{s_{i+1}+1}$ satisfies the condition in (A2), and

$$
\begin{aligned}
B\left(\mathbf{d}_{1}^{s_{i+1}+1} ; i+2\right) & =d_{s_{i+1}+1}+B\left(\mathbf{d}_{1}^{s_{i+1}} ; i+1\right) \\
& =d_{s_{i+1}+1}+d_{s_{1}}+d_{s_{2}}+\cdots+d_{s_{i}}+d_{s_{i+1}},
\end{aligned}
$$

where $B\left(\mathbf{d}_{1}^{s_{i+1}} ; i+1\right)=d_{s_{1}}+d_{s_{2}}+\cdots+d_{s_{i}}+d_{s_{i+1}}$ follows from the induction hypothesis. We have completed the induction and the theorem is proved.

\section{An Optimal Construction is a Greedy CONSTRUCTION}

For $M \geq 1$ and $1 \leq k \leq M$, let $B(M, k)$ be the the largest possible maximum representable integer $B\left(\mathbf{d}_{1}^{M} ; k\right)$ over all $\mathbf{d}_{1}^{M} \in \mathcal{A}_{M}$, i.e.,

$$
B(M, k)=\max _{\mathbf{d}_{1}^{M} \in \mathcal{A}_{M}} B\left(\mathbf{d}_{1}^{M} ; k\right) .
$$

We call the construction of an $M$-sequence $\mathbf{d}_{1}^{* M} \in \mathcal{A}_{M}$ an optimal construction if $B(M, k)=B\left(\mathbf{d}_{1}^{* M} ; k\right)$.

In the following, we will show that every optimal construction is a greedy construction. Note that for $M=1$, the only sequence satisfying the condition in (A2) is $d_{1}=1$, which is also the only sequence generated by (5) as we have shown in Section III. As there is only one construction in this case, the optimal construction is also the greedy construction. For $M \geq 2$ and $k=M$, it is easy to see that the optimal construction is given by $d_{1}^{*}=1, d_{2}^{*}=2, d_{3}^{*}=2^{2}, \ldots$, $d_{M}^{*}=2^{M-1}$. In this case, we have shown in Section III that $d_{1}=1, d_{2}=2, d_{3}=2^{2}, \ldots, d_{M}=2^{M-1}$ is the only possible sequence generated by (5), and it then follows that the optimal construction is also the greedy construction. As such, in the following theorem we only consider the nontrivial case with $M \geq 2$ and $1 \leq k \leq M-1$, and show that every optimal construction is a greedy construction. 
Theorem 9 Let $M \geq 2$ and $1 \leq k \leq M-1$. Suppose that $B(M, k)=B\left(\mathbf{d}_{1}^{* M} ; k\right)$ for some $\mathbf{d}_{1}^{* \bar{M}} \in \mathcal{A}_{M}$. Then $\mathbf{d}_{1}^{* M} \in$ $\mathcal{G}_{M, k}$. In other words, every optimal construction is a greedy construction.

Theorem 5 implies that an optimal construction $\mathbf{d}_{1}^{* M}$ in Figure 1 that achieves the maximum buffer size among $\left\{B\left(\mathbf{d}_{1}^{M} ; k\right): \mathbf{d}_{1}^{M} \in \mathcal{A}_{M}\right\}$ could be given by a greedy construction, namely, $\mathbf{d}_{1}^{* M} \in \mathcal{G}_{M, k}$. From [15], we know that the size of $\mathcal{A}_{M}$ grows at least with $2^{M-1}$ (exponential growth). Also, it is easy to see that the number of $k$-sequences $\left(n_{1}, n_{2}, \ldots, n_{k}\right)$ with $n_{1} \geq 2, n_{2} \geq 1, \ldots, n_{k} \geq 1$ and $\sum_{i=1}^{k} n_{i}=M$ is given by $\left(\begin{array}{c}M-2 \\ k-1\end{array}\right)$. It follows that the size of $\mathcal{G}_{M, k}$ grows at most with $(M-2)^{k-1}$ (polynomial growth). As such, the complexity of searching for an optimal construction can be greatly reduced from exponential time to polynomial time by only considering the greedy constructions instead of performing an exhaustive search.

Proof. (Proof of Theorem 9) We divide the proof into five parts.

(i) First we show that $B(M, k) \geq d_{M}^{*}$. Suppose on the contrary that $B(M, k)<d_{M}^{*}$. Clearly, $B(M, k)=B\left(\mathbf{d}^{* M} ; k\right) \geq$ $1=d_{1}^{*}$. Therefore, we have $d_{1}^{*} \leq B\left(\mathbf{d}_{1}^{* M} ; k\right)<d_{M}^{*}$ and it follows that there exists an $\ell^{\prime}$, where $1 \leq \ell^{\prime} \leq M-1$, such that $d_{\ell^{\prime}}^{*} \leq B(M, k)<d_{\ell^{\prime}+1}^{*}$.

Let $h_{\ell}=d_{\ell}^{*}$ for $\ell=1,2, \ldots, \ell^{\prime}$, and $h_{\ell}=B(M, k)+\ell-\ell^{\prime}$ for $\ell=\ell^{\prime}+1, \ell^{\prime}+2, \ldots, M$. We claim that $\mathbf{h}_{1}^{M} \in \mathcal{A}_{M}$. Since $\mathbf{d}_{1}^{* M} \in \mathcal{A}_{M}$, we have $d_{1}^{*}=1$ and $d_{\ell}^{*} \leq d_{\ell+1}^{*} \leq 2 d_{\ell}^{*}$ for $\ell=1,2, \ldots, M-1$. As such, it follows from $h_{\ell}=d_{\ell}^{*}$ for $\ell=1,2, \ldots, \ell^{\prime}$ that $h_{1}=1$ and $h_{\ell} \leq h_{\ell+1} \leq 2 h_{\ell}$ for $\ell=1,2, \ldots, \ell^{\prime}-1$. From $d_{\ell^{\prime}}^{*} \leq B(M, k)<d_{\ell^{\prime}+1}^{*}$ and $d_{\ell^{\prime}+1}^{*} \leq$ $2 d_{\ell^{\prime}}^{*}$, we have

$$
\begin{aligned}
& h_{\ell^{\prime}+1}=B(M, k)+1 \geq d_{\ell^{\prime}}^{*}+1>d_{\ell^{\prime}}^{*}=h_{\ell^{\prime}}, \\
& h_{\ell^{\prime}+1}=B(M, k)+1 \leq d_{\ell^{\prime}+1}^{*} \leq 2 d_{\ell^{\prime}}^{*}=2 h_{\ell^{\prime}} .
\end{aligned}
$$

This leads to $h_{\ell^{\prime}} \leq h_{\ell^{\prime}+1} \leq 2 h_{\ell^{\prime}}$. It is also easy to see from $h_{\ell}=B(M, k)+\ell-\ell^{\prime}$ for $\ell=\ell^{\prime}+1, \ell^{\prime}+2, \ldots, M$ that $h_{\ell+1}=h_{\ell}+1$ for $\ell=\ell^{\prime}+1, \ell^{\prime}+2, \ldots, M-1$, and hence $h_{\ell} \leq h_{\ell+1} \leq 2 h_{\ell}$ for $\ell=\ell^{\prime}+1, \ell^{\prime}+2, \ldots, M-1$.

From $d_{\ell^{\prime}}^{*} \leq B(M, k)=B\left(\mathbf{d}_{1}^{* M} ; k\right)<d_{\ell^{\prime}+1}^{*}$ for some $1 \leq$ $\ell^{\prime} \leq M-1$, Lemma 6 , and $h_{\ell}=d_{\ell}^{*}$ for $\ell=1,2, \ldots, \ell^{\prime}$, we have

$$
B(M, k)=B\left(\mathbf{d}_{1}^{* M} ; k\right)=B\left(\mathbf{d}_{1}^{* \ell^{\prime}} ; k\right)=B\left(\mathbf{h}_{1}^{\ell^{\prime}} ; k\right) .
$$

It follows that the integers $0,1,2, \ldots, B(M, k)$ are representable with at most $k$ of the $\ell^{\prime}$ integers $h_{1}, h_{2}, \ldots, h_{\ell^{\prime}}$ by using the $\mathcal{C}$-transform. As $B(M, k)<h_{\ell^{\prime}+1}=B(M, k)+1$ and $h_{\ell^{\prime}+1} \leq h_{\ell^{\prime}+2} \leq \cdots \leq h_{M}, h_{\ell^{\prime}+1}, h_{\ell^{\prime}+2}, \ldots, h_{M}$ will not be used in the representation of the integers $0,1, \ldots, B(M, k)$ by using the $\mathcal{C}$-transform. As such, we have $B\left(\mathbf{h}_{1}^{M} ; k\right) \geq$ $B(M, k)$. Furthermore, the integer $x$, where $B(M, k)+1 \leq$ $x \leq B(M, k)+M-\ell^{\prime}$, is representable by $h_{x-B(M, k)+\ell^{\prime}}=$ $\left(x-B(M, k)+\ell^{\prime}\right)+B(M, k)-\ell^{\prime}=x$ as $\ell^{\prime}+1 \leq$ $x-B(M, k)+\ell^{\prime} \leq M$. Combining with $B\left(\mathbf{h}_{1}^{M} ; k\right) \geq B(M, k)$ leads to $B\left(\mathbf{h}_{1}^{M} ; k\right) \geq B(M, k)+M-\ell^{\prime}>B(M, k)$.
As a result, we have

$$
B(M, k)=\max _{\mathbf{d}_{1}^{M} \in \mathcal{A}_{M}} B\left(\mathbf{d}_{1}^{M} ; k\right) \geq B\left(\mathbf{h}_{1}^{M} ; k\right)>B(M, k),
$$

and we have reached a contradiction.

(ii) From $B(M, k)=B\left(\mathbf{d}_{1}^{* M} ; k\right) \geq d_{M}^{*}$ in (i) and Lemma 6 , we have $B\left(\mathbf{d}_{1}^{* M} ; k\right)=d_{M}^{*}+B\left(\mathbf{d}_{1}^{* M-1} ; k-1\right)$. Let $s_{k}=M$, then we have

$$
B\left(\mathbf{d}_{1}^{* s_{k}} ; k\right)=d_{s_{k}}^{*}+B\left(\mathbf{d}_{1}^{* s_{k}-1} ; k-1\right) .
$$

Let

$s_{k-1}=\max \left\{1 \leq \ell \leq s_{k}-1: d_{\ell}^{*} \leq B\left(\mathbf{d}_{1}^{* s_{k}-1} ; k-1\right)\right\}$,

then it follows from Lemma 6 that

$$
\begin{aligned}
B\left(\mathbf{d}_{1}^{* s_{k}-1} ; k-1\right) & =B\left(\mathbf{d}_{1}^{* s_{k}-2} ; k-1\right) \\
& \vdots \\
& =B\left(\mathbf{d}_{1}^{* s_{k-1}} ; k-1\right) \\
& =d_{s_{k-1}}^{*}+B\left(\mathbf{d}_{1}^{* s_{k-1}-1} ; k-2\right) .
\end{aligned}
$$

By repeating the above procedure for $k-1$ times, we have

$$
\begin{aligned}
B\left(\mathbf{d}_{1}^{* s_{i+1}-1} ; i\right) & =B\left(\mathbf{d}_{1}^{* s_{i+1}-2} ; i\right)=\cdots=B\left(\mathbf{d}_{1}^{* s_{i}} ; i\right) \\
& =d_{s_{i}}^{*}+B\left(\mathbf{d}_{1}^{* s_{i}-1} ; i-1\right),
\end{aligned}
$$

where

$$
s_{i}=\max \left\{1 \leq \ell \leq s_{i+1}-1: d_{\ell}^{*} \leq B\left(\mathbf{d}_{1}^{* s_{i+1}-1} ; i\right)\right\},
$$

for $i=1,2, \ldots, k-1$. From (20) and (21), it is easy to deduce by induction on $i$ that

$$
B\left(\mathbf{d}_{1}^{* s_{i}} ; i\right)=d_{s_{1}}^{*}+d_{s_{2}}^{*}+\cdots+d_{s_{i}}^{*}, i=1,2, \ldots, k .
$$

(iii) We claim that $s_{1} \geq 2$. Suppose on the contrary that $s_{1}=1$. We will show by induction on $i$ that $s_{i}=i$ for all $i=1,2, \ldots, k$. As such, it follows from $k \leq M-1$ that $s_{k}=k \neq M$, and we have reached a contradiction.

Assume that $s_{1}=1, s_{2}=2, \ldots, s_{i}=i$ for some $1 \leq i \leq$ $k-1$. Note that $s_{i+1} \geq s_{i}+1=i+1$. Suppose that $s_{i+1} \geq$ $i+2$. Then $s_{i+1}-1 \geq i+1$ and $d_{i+1}^{*}, d_{i+2}^{*}, \ldots, d_{s_{i+1}-1}^{*}$ will not be used in the representation of an integer less than $d_{i+1}^{*}$ by using the $\mathcal{C}$-transform. As $d_{1}^{*}=1$ and $d_{\ell}^{*} \leq d_{\ell+1}^{*} \leq 2 d_{\ell}^{*}$ for $\ell=1,2, \ldots, M-1$, we have

$$
\begin{aligned}
d_{i+1}^{*} & \leq 2 d_{i}^{*}=d_{i}^{*}+d_{i}^{*} \\
& \leq d_{i}^{*}+2 d_{i-1}^{*}=d_{i}^{*}+d_{i-1}^{*}+d_{i-1}^{*} \\
& \leq d_{i}^{*}+d_{i-1}^{*}+2 d_{i-2}^{*} \\
& \vdots \\
& \leq d_{i}^{*}+d_{i-1}^{*}+\cdots+d_{2}^{*}+2 d_{1}^{*} \\
& =B\left(\mathbf{d}^{* i}{ }_{1} ; i\right)+1,
\end{aligned}
$$

where the equality follows from (23) and $s_{1}=1, s_{2}=2, \ldots$, $s_{i}=i$. This implies that the integers $0,1, \ldots, d_{i+1}^{*}-1$ are representable by using at most $i$ of the $s_{i+1}-1$ integers $d_{1}^{*}, d_{2}^{*}, \ldots, d_{s_{i+1}-1}^{*}$ by using the $\mathcal{C}$-transform. Furthermore, $d_{i+1}^{*}$ is clearly representable, implying that $B\left(\mathbf{d}_{1}^{* s_{i+1}-1} ; i\right) \geq$ 
$d_{i+1}^{*}$. As a result, we have from (22) that $s_{i} \geq i+1$, contradicting to the induction hypothesis that $s_{i}=i$. Therefore, we must have $s_{i+1}=i+1$, and the induction is completed.

(iv) Let $s_{0}=0$ and $n_{i}=s_{i}-s_{i-1}$ for $i=1,2, \ldots, k$, and let $d_{1}, d_{2}, \ldots, d_{M}$ be the sequence generated by $n_{1}, n_{2}, \ldots, n_{k}$ by using (5). In the following, we show by induction on $\ell$ that $d_{\ell}^{*} \leq d_{\ell}$ for all $\ell=1,2, \ldots, M$, and $d_{\ell}^{*}<d_{\ell}$ for all $\ell=\ell^{\prime}, \ell^{\prime}+1, \ldots, M$ if $d_{\ell^{\prime}}^{*}<d_{\ell^{\prime}}$ for some $2 \leq \ell^{\prime} \leq M$ (note that $d_{1}^{*}=d_{1}=1$ ).

From the definition of $B\left(\mathbf{d}_{1}^{* s_{2}-1} ; 1\right)$, it is not difficult to see that $B\left(\mathbf{d}_{1}^{* s_{2}-1} ; 1\right)=d_{\ell^{\prime}}^{*}$, where

$$
\begin{array}{r}
\ell^{\prime}=\max \left\{2 \leq \ell \leq s_{2}-1: d_{2}^{*}-d_{1}^{*} \leq 1, d_{3}^{*}-d_{2}^{*} \leq 1, \ldots,\right. \\
\left.d_{\ell}^{*}-d_{\ell-1}^{*} \leq 1\right\} .(24)
\end{array}
$$

In other words, $\ell^{\prime}$ is the largest index in $\left\{2,3, \ldots, s_{2}-1\right\}$ such that the difference between $d_{\ell}^{*}$ and $d_{\ell-1}^{*}$ is at most one for all $\ell=2,3, \ldots, \ell^{\prime}$. (Note that $\ell^{\prime}$ is well defined as we have $s_{2}-1 \geq s_{1} \geq 2$ from (iii) and $d_{2}^{*}-d_{1}^{*} \leq 2 d_{1}^{*}-d_{1}^{*}=d_{1}^{*}=1$.) If $2 \leq \ell^{\prime} \leq s_{2}-2$, then $d_{\ell^{\prime}+1}^{*}-d_{\ell^{\prime}}^{*} \geq 2$ and hence $d_{\ell^{\prime}+1}^{*} \geq$ $d_{\ell^{\prime}}^{*}+2=B\left(\mathbf{d}_{1}^{* s_{2}-1} ; 1\right)+2>B\left(\mathbf{d}_{1}^{* s_{2}-1} ; 1\right)$. On the other hand, we have $B\left(\mathbf{d}_{1}^{* s_{2}-1} ; 1\right)=d_{s_{2}-1}^{*}$ if $\ell^{\prime}=s_{2}-1$. As such, it follows from (22) that $s_{1}=\ell^{\prime}$. As $d_{1}^{*}=1$ and the difference between $d_{\ell}^{*}$ and $d_{\ell-1}^{*}$ is at most one for $\ell=2,3, \ldots, s_{1}$, we then have from $d_{\ell}=\ell$ for $\ell=1,2, \ldots, s_{1}$ in (6) that

$$
d_{\ell}^{*} \leq \ell=d_{\ell}, \ell=1,2, \ldots, s_{1} .
$$

Furthermore, if $d_{\ell^{\prime}}^{*}<d_{\ell^{\prime}}=\ell^{\prime}$ for some $2 \leq \ell^{\prime} \leq s_{1}$, then

$$
d_{\ell}^{*} \leq d_{\ell^{\prime}}^{*}+\left(\ell-\ell^{\prime}\right)<\ell^{\prime}+\left(\ell-\ell^{\prime}\right)=\ell=d_{\ell},
$$

for all $\ell=\ell^{\prime}, \ell^{\prime}+1, \ldots, s_{1}$.

We have proved in (25) and (26) that $d_{1}^{*} \leq d_{1}, d_{2}^{*} \leq d_{2}, \ldots$, $d_{s_{1}}^{*} \leq d_{s_{1}}$, and $d_{\ell^{\prime}}^{*}<d_{\ell^{\prime}}, d_{\ell^{\prime}+1}^{*}<d_{\ell^{\prime}+1}, \ldots, d_{s_{1}}^{*}<d_{s_{1}}$ if $d_{\ell^{\prime}}^{*}<d_{\ell^{\prime}}$ for some $2 \leq \ell^{\prime} \leq s_{1}$. Now assume as the induction hypothesis that $d_{1}^{*} \leq d_{1}, d_{2}^{*} \leq d_{2}, \ldots, d_{\ell}^{*} \leq d_{\ell}$ for some $s_{1} \leq \ell \leq M-1$, and $d_{\ell^{\prime}}^{*}<d_{\ell^{\prime}}, d_{\ell^{\prime}+1}^{*}<d_{\ell^{\prime}+1}, \ldots, d_{\ell}^{*}<d_{\ell}$ if $d_{\ell^{\prime}}^{*}<d_{\ell^{\prime}}$ for some $2 \leq \ell^{\prime} \leq \ell$. We then consider the following two cases:

Case 1. $\ell=s_{i}$, where $1 \leq i \leq k-1$ : As $\mathbf{d}_{1}^{* M} \in \mathcal{A}_{M}$, we have $d_{\ell+1}^{*} \leq 2 d_{\ell}^{*}$. In this case, we have from (7) that $d_{\ell+1}=d_{s_{i}+1}=2 d_{s_{i}}=2 d_{\ell}$. It then follows from the induction hypothesis that

$$
d_{\ell+1}^{*} \leq 2 d_{\ell}^{*} \leq 2 d_{\ell}=d_{\ell+1} .
$$

Furthermore, if $d_{\ell^{\prime}}^{*}<d_{\ell^{\prime}}$ for some $2 \leq \ell^{\prime} \leq \ell$, then we have $d_{\ell^{\prime}}^{*}<d_{\ell^{\prime}}, d_{\ell^{\prime}+1}^{*}<d_{\ell^{\prime}+1}, \ldots, d_{\ell}^{*}<d_{\ell}$ from the induction hypothesis. As such, it follows that

$$
d_{\ell+1}^{*} \leq 2 d_{\ell}^{*}<2 d_{\ell}=d_{\ell+1} .
$$

Case 2. $\ell=s_{i}+j$, where $1 \leq i \leq k-1$ and $1 \leq j \leq$ $n_{i+1}-1$ : Consider an integer $x$, where $0 \leq x \leq d_{s_{i}+j+1}^{*}-1$. As $s_{i}+j+1 \leq s_{i}+n_{i+1}=s_{i+1}$, we have from (23) that

$$
\begin{aligned}
x & \leq d_{s_{i}+j+1}^{*}-1 \leq d_{s_{i+1}}^{*}-1 \\
& \leq d_{s_{1}}^{*}+d_{s_{2}}^{*}+\cdots+d_{s_{i+1}}^{*} \\
& =B\left(\mathbf{d}_{1}^{* s_{i+1}} ; i+1\right) .
\end{aligned}
$$

As such, all the integers $0,1, \ldots, d_{s_{i}+j+1}^{*}-1$ are representable by using at most $i+1$ of the $s_{i+1}$ integers $d_{1}^{*}, d_{2}^{*}, \ldots, d_{s_{i+1}}^{*}$ by using the $\mathcal{C}$-transform.

We claim that

$$
d_{s_{i}+j+1}^{*}-1 \leq d_{s_{i}+j}^{*}+B\left(\mathbf{d}_{1}^{* s_{i}} ; i\right) .
$$

If $d_{s_{i}+j+1}^{*}-1<d_{s_{i}+j}^{*}$, then there is nothing to prove. On the other hand, if $d_{s_{i}+j+1}^{*}-1 \geq d_{s_{i}+j}^{*}$, then consider an integer $x$, where $d_{s_{i}+j}^{*} \leq x \leq d_{s_{i}+j+1}^{*}-1$. According to the $\mathcal{C}$ transform, $d_{s_{i}+j+1}^{*}, d_{s_{i}+j+2}^{*}, \ldots, d_{s_{i+1}}^{*}$ will not be used in the representation of $x$, but $d_{s_{i}+j}^{*}$ will be used. As a result, the integer $x-d_{s_{i}+j}^{*}$, where $0 \leq x-d_{s_{i}+j}^{*} \leq d_{s_{i}+j+1}^{*}-d_{s_{i}+j}^{*}-1$, is representable by using at most $i$ of the $s_{i}+j-1$ integers $d_{1}^{*}, d_{2}^{*}, \ldots, d_{s_{i}+j-1}^{*}$ by using the $\mathcal{C}$-transform. This implies that

$$
d_{s_{i}+j+1}^{*}-d_{s_{i}+j}^{*}-1 \leq B\left(\mathbf{d}_{1}^{* s_{i}+j-1} ; i\right)=B\left(\mathbf{d}_{1}^{* s_{i}} ; i\right),
$$

where $B\left(\mathbf{d}_{1}^{* s_{i}+j-1} ; i\right)=B\left(\mathbf{d}_{1}^{* s_{i}} ; i\right)$ follows from (21).

From (27), (23), and the induction hypothesis, we have

$$
\begin{aligned}
d_{s_{i}+j+1}^{*} & \leq d_{s_{i}+j}^{*}+B\left(\mathbf{d}_{1}^{* s_{i}} ; i\right)+1 \\
& =d_{s_{i}+j}^{*}+d_{s_{1}}^{*}+d_{s_{2}}^{*}+\cdots+d_{s_{i}}^{*}+1 \\
& \leq d_{s_{i}+j}+d_{s_{1}}+d_{s_{2}}+\cdots+d_{s_{i}}+1 .
\end{aligned}
$$

As $1 \leq j \leq n_{i+1}-1$, we have $2 \leq j+1 \leq n_{i+1}$. It then follows from (28) and (7) that

$$
\begin{aligned}
d_{\ell+1}^{*}= & d_{s_{i}+j+1}^{*} \\
\leq & d_{s_{i}+j}+d_{s_{1}}+d_{s_{2}}+\cdots+d_{s_{i}}+1 \\
= & 2 d_{s_{i}}+(j-1)\left(d_{s_{1}}+d_{s_{2}}+\cdots+d_{s_{i}}+1\right)+d_{s_{1}} \\
& +d_{s_{2}}+\cdots+d_{s_{i}}+1 \\
= & 2 d_{s_{i}}+j\left(d_{s_{1}}+d_{s_{2}}+\cdots+d_{s_{i}}+1\right) \\
= & d_{s_{i}+j+1}=d_{\ell+1} .
\end{aligned}
$$

Furthermore, if $d_{\ell^{\prime}}^{*}<d_{\ell^{\prime}}$ for some $2 \leq \ell^{\prime} \leq \ell$, then we have $d_{\ell^{\prime}}^{*}<d_{\ell^{\prime}}, d_{\ell^{\prime}+1}^{*}<d_{\ell^{\prime}+1}, \ldots, d_{\ell}^{*}<d_{\ell}$ from the induction hypothesis. As such, the inequality in (28) becomes a strict inequality and it then follows that

$$
d_{\ell+1}^{*}<d_{\ell+1} .
$$

(v) Finally, we show that $d_{\ell}^{*}=d_{\ell}$ for all $\ell=1,2, \ldots, M$. From (23) and $d_{\ell}^{*} \leq d_{\ell}$ for all $\ell=1,2, \ldots, M$ in (iv), we have

$$
\begin{aligned}
B(M, k) & =B\left(\mathbf{d}_{1}^{* s_{k}} ; k\right)=d_{s_{1}}^{*}+d_{s_{2}}^{*}+\cdots+d_{s_{k}}^{*} \\
& \leq d_{s_{1}}+d_{s_{2}}+\cdots+d_{s_{k}} .
\end{aligned}
$$

From the definition of $B(M, k)$ in (19) and from Theorem 5, we have

$$
\begin{aligned}
B(M, k) & =\max _{\mathbf{h}_{1}^{M} \in \mathcal{A}_{M}} B\left(\mathbf{h}_{1}^{M} ; k\right) \\
& \geq B\left(\mathbf{d}_{1}^{M} ; k\right)=d_{s_{1}}+d_{s_{2}}+\cdots+d_{s_{k}} .
\end{aligned}
$$

As such, it follows from (29) and (30) that $B(M, k)=d_{s_{1}}+$ $d_{s_{2}}+\cdots+d_{s_{k}}$ and $d_{s_{i}}^{*}=d_{s_{i}}$ for all $i=1,2, \ldots, k$. This leads to $d_{\ell}^{*}=d_{\ell}$ for all $\ell=1,2, \ldots, M$. Otherwise, we must have $d_{\ell^{\prime}}^{*}<d_{\ell^{\prime}}$ for some $2 \leq \ell^{\prime} \leq M$, and it follows from the results 
in (iv) that $d_{\ell}^{*}<d_{\ell}$ for all $\ell=\ell^{\prime}, \ell^{\prime}+1, \ldots, M$. In particular, we have $d_{s_{k}}^{*}=d_{M}^{*}<d_{M}=d_{s_{k}}$, and a contradiction is reached. This shows that $\mathbf{d}_{1}^{* M}=\mathbf{d}_{1}^{M} \in \mathcal{G}_{M, k}$ and we have completed the proof that every optimal construction is a greedy construction.

\section{CONCLUSiON}

In this paper, we considered the constructions of optical queues by using optical Switches and fiber Delay Lines (SDL) with a limited number of recirculations through the fibers. Such a limitation on the number of recirculations comes from practical feasibility considerations, such as crosstalk, power loss, amplified spontaneous emission (ASE) from the Erbium doped fiber amplifiers (EDFA), and the pattern effect of the optical switches.

We first transformed the design of the fiber delays in such SDL constructions to an equivalent integer representation problem. We then gave a class of greedy constructions for the $M$-sequence $\mathbf{d}_{1}^{M}$ in the equivalent integer representation problem, and obtained an explicit recursive expression for such an $M$-sequence $\mathbf{d}_{1}^{M}$ and for the corresponding maximum representable integer $B\left(\mathbf{d}_{1}^{M} ; k\right)$. Finally, we showed that an optimal construction that achieves the largest possible maximum representable integer can be given by a greedy construction. The results can be applied to the constructions of optical 2-to1 FIFO multiplexers with a limited number of recirculations, and we showed that the complexity of searching for an optimal construction can be greatly reduced from exponential time to polynomial time by only considering the greedy constructions instead of performing an exhaustive search. Similar results can be obtained for linear compressors and linear decompressors with a limited number of recirculations.

\section{REFERENCES}

[1] M. J. Karol, "Shared-memory optical packet (ATM) switch," in Proceedings SPIE : Multigigabit Fiber Communication Systems (1993), October 1993, vol. 2024, pp. 212-222.

[2] I. Chlamtac, A. Fumagalli, L. G. Kazovsky, P. Melman, W. H. Nelson, P. Poggiolini, M. Cerisola, A. N. M. M. Choudhury, T. K. Fong, R. T. Hofmeister, C.-L. Lu, A. Mekkittikul, D. J. M. Sabido IX, C.-J. Suh, and E. W. M. Wong, "Cord: contention resolution by delay lines," IEEE Journal on Selected Areas in Communications, vol. 14, pp. 1014-1029, June 1996

[3] I. Chlamtac, A. Fumagalli, and C.-J. Suh, "Multibuffer delay line architectures for efficient contention resolution in optical switching nodes," IEEE Transactions on Communications, vol. 48, pp. 2089-2098, December 2000.

[4] J. T. Tsai, "COD: architectures for high speed time-based multiplexers and buffered packet switches," Ph.D. Dissertation, University of California, San Diego, La Jolla, CA, USA, 1995.

[5] R. L. Cruz and J.-T. Tsai, "COD: alternative architectures for high speed packet switching," IEEE/ACM Transactions on Networking, vol. 4, pp. 11-21, February 1996.

[6] D. K. Hunter, D. Cotter, R. B. Ahmad, D. Cornwell, T. H. Gilfedder, P. J. Legg, and I. Andonovic, " $2 \times 2$ buffered switch fabrics for traffic routing, merging and shaping in photonic cell networks," IEEE Journal of Lightwave Technology, vol. 15, pp. 86-101, January 1997.

[7] D. K. Hunter, W. D. Cornwell, T. H. Gilfedder, A. Franzen, and I. Andonovic, "SLOB: a switch with large optical buffers for packet switching," IEEE Journal of Lightwave Technology, vol. 16, pp. 17251736, October 1998.

[8] E. A. Varvarigos, "The "packing" and the "scheduling packet" switch architectures for almost all-optical lossless networks," IEEE Journal of Lightwave Technology, vol. 16, pp. 1757-1767, October 1998.
[9] D. K. Hunter and I. Andonovic, "Approaches to optical Internet packet switching," IEEE Communications Magazine, vol. 38, pp. 116-122, September 2000.

[10] S. Yao, B. Mukherjee, and S. Dixit, "Advances in photonic packet switching: An overview," IEEE Communications Magazine, vol. 38, pp. 84-94, February 2000.

[11] C.-S. Chang, D.-S. Lee, and C.-K. Tu, "Recursive construction of FIFO optical multiplexers with switched delay lines," IEEE Transactions on Information Theory, vol. 50, pp. 3221-3233, December 2004.

[12] C.-S. Chang, D.-S. Lee, and C.-K. Tu, "Using switched delay lines for exact emulation of FIFO multiplexers with variable length bursts," IEEE Journal on Selected Areas in Communications, vol. 24, pp. 108-117, April 2006.

[13] C.-C. Chou, C.-S. Chang, D.-S. Lee and J. Cheng, "A necessary and sufficient condition for the construction of 2-to-1 optical FIFO multiplexers by a single crossbar switch and fiber delay lines," IEEE Transactions on Information Theory, vol. 52, pp. 4519-4531, October 2006.

[14] Y.-T. Chen, C.-S. Chang, J. Cheng, and D.-S. Lee, "Feedforward SDL constructions of output-buffered multiplexers and switches with variable length bursts," in Proceedings IEEE International Conference on Computer Communications (INFOCOM'07), Anchorage, AK, USA, May 6-12, 2007.

[15] J. Cheng, "Constructions of fault tolerant optical 2-to-1 FIFO multiplexers," IEEE Transactions on Information Theory, vol. 53, November 2007.

[16] J. Cheng, "Constructions of optical 2-to-1 FIFO multiplexers with a limited number of recirculations," submitted to IEEE Transactions on Information Theory, 2007.

[17] C.-S. Chang, Y.-T. Chen, and D.-S. Lee, "Constructions of optical FIFO queues," IEEE Transactions on Information Theory, vol. 52, pp. 28382843, June 2006.

[18] A. Bouillard and C.-S. Chang, "An explicit control algorithm for optical FIFO queues," submitted to IEEE Transactions on Information Theory, 2007.

[19] P.-K. Huang, C.-S. Chang, J. Cheng, and D.-S. Lee, "Recursive constructions of parallel FIFO and LIFO queues with switched delay lines," IEEE Transactions on Information Theory, vol. 53, pp. 1778-1798, May 2007.

[20] A. D. Sarwate and V. Anantharam, "Exact emulation of a priority queue with a switch and delay lines," Queueing Systems: Theory and Applications, vol. 53, pp. 115-125, July 2006.

[21] H.-C. Chiu, C.-S. Chang, J. Cheng, and D.-S. Lee, "A simple proof for the constructions of optical priority queues," Queueing Systems: Theory and Applications, vol. 56, June 2007

[22] H.-C. Chiu, C.-S. Chang, J. Cheng, and D.-S. Lee, "Using a single switch with $O(M)$ inputs/outputs for the construction of an optical priority queue with $O\left(M^{3}\right)$ buffer," in Proceedings IEEE International Conference on Computer Communications (INFOCOM'07 Minisymposium), Anchorage, AK, USA, May 6-12, 2007.

[23] Y.-T. Chen, J. Cheng, and D.-S. Lee, "Multistage constructions of linear compressors, non-overtaking delay lines, and flexible delay lines," submitted to IEEE/ACM Transactions on Networking 2007. Conference version in Proceedings IEEE International Conference on Computer Communications (INFOCOM'06), Barcelona, Spain, April 23-29, 2006.

[24] C.-S. Chang, T.-H. Chao, J. Cheng, and D.-S. Lee, "Constructions of fault tolerant linear compressors and linear decompressors," in Proceedings IEEE International Conference on Computer Communications (INFOCOM'07), Anchorage, AK, USA, May 6-12, 2007.

[25] S.-Y. R. Li and X. J. Tan, "Fiber memory," submitted to IEEE Transactions on Information Theory, 2006.

[26] E. F. Burmeister and J. E. Bowers, "Integrated gate matrix switch for optical packet buffering," IEEE Photonics Technology Letters, vol. 18 , pp. 103-105, January 2006.

[27] C. P. Larsen and M. Gustavsson, "Linear crosstalk in $4 \times 4$ semiconductor optical amplifier gate switch matrix," IEEE Journal of Lightwave Technology, vol. 15, pp. 1865-1870, October 1997.

[28] R. Geldenhuys, Z. Wang, N. Chi, I. Tafur Monroy, A. M. J. Koonen, H. J. S. Dorren, F. W. Leuschner, G. D. Khoe, and S. Yu, "Multiple recirculations through Crosspoint switch fabric for recirculating optical buffering," Electronics Letters, vol. 41, pp. 1136-1137, September 2005.

[29] J. Cheng, C.-S. Chang, T.-H. Chao, D.-S. Lee, and C.-M. Lien, "On constructions of optical queues with a limited number of recirculations," Technical Report, National Tsing Hua University, Hsinchu, Taiwan, R.O.C., June 2007. Available from http://www.ee.nthu.edu.tw/ cschang/optical-queues-LR.pdf. 